\title{
LA TRASLACIÓN DE LA MODULACIÓN \\ Y LAS PROPORCIONES EN ARQUITECTURA. \\ DE VITRUVIO A VILLARD DE HONNECOURT. \\ EL CASO DE SANTA MAGDALENA D'EMPÚRIES
}

\author{
Montse Valls Mora* \\ Universitat de Barcelona \\ mvallsmora@gmail.com
}

Los conocimientos que se utilizan para el diseño de los edificios eclesiásticos, durante la antigüedad tardía y el alto medioevo, ¿son producto de una translatio? Los métodos y herramientas para formalizar lo que entendemos por espacio arquitectónico que aplica un arquitecto romano como Vitruvio, ¿son los mismos que maneja Villard de Honnecourt en época medieval?

La respuesta a esta cuestión la he desarrollado siguiendo dos líneas de análisis: el saber teórico y la práctica aplicada, durante la cronología estudiada. El conocimiento teórico es de tipo contextual. Describo aspectos del entorno cultural, relacionados con la concepción teórico-formal de la arquitectura y sus herramientas. Mientras que el examen pragmático lo centro en un ejemplo concreto, el estudio geométrico-modular de Santa Magdalena d’Empúries.

DE LA CONCEPCIÓN ARQUITECTÓNICA Y LA IDEA DE BELLEZA: GEOMETRÍA, MÓDULOS Y PROPORCIÓN

Para diseñar la forma de los espacios, la arquitectura se sirve inicialmente de conceptos geométricos, módulos de medición y proporciones. Estas tres herramientas han sido y son la base de cualquier proyecto arquitectónico. Sin embargo, el uso de unas u otras variantes, no tiene por qué ser constante en el tiempo. En el caso de la geometría se trata de conocimientos teóricos aplicados y se concreta en el empleo de las diferentes tipologías poligonales y las propiedades aritméticas que

\footnotetext{
* Equip de Recerca Arqueològica i Arqueomètrica de la Universitat de Barcelona (ERAAUB), Corpus Architecturae Religiosae Europeae (saec. IV-X) (CARE-Hispania), y El impacto de la arquitectura religiosa en el paisaje urbano y rural del nordeste de Hispania y las Islas Baleares (ss. IV-X d.C.) (HAR2012-35177/Hist).
} 
de ellas se derivan. Los módulos son la normalización de magnitudes de medición y, si bien se basan en la codificación romana, se pueden producir modificaciones que los distancien sensiblemente de las originales, o se hace patente la utilización de unos y la decadencia de otros. Finalmente la proporción o razón entre las partes no es en sí misma una herramienta ya que responde a la combinación de las dos anteriores, es un factor complejo basado en la combinación de leyes aritmético-geométricas-modulares provenientes de la semejanza euclidiana y los conceptos de armonía y escala.

Es indudable que hay otros factores decisivos en el diseño del proyecto de arquitectura como el emplazamiento, el programa de uso, el presupuesto del que se dispone, o los objetivos del promotor, pero no entran en los objetivos de este trabajo.

Otro elemento que influye en la génesis de la arquitectura, y que hay que entender como contexto cultural, es la interpretación de la 'belleza'. Cada cultura ha tenido sus paradigmas y tratar de entenderlos y definirlos nos acerca al pensamiento estético sobre su arquitectura. En este sentido quiero destacar como inicialmente Platón se sirve de la geometría para expresar el significado de la perfección en la belleza². Siglos después, Plotino distinguirá dos tipos de belleza: la formal platónica basada en la proporción y pureza de las formas como consecuencia de la razón y la espiritual a través del intelecto y como unidad compleja ${ }^{3}$. Ésta última, la "belleza del alma", cobrará auge y se desarrolla en un conjunto de corrientes filosófico-religiosas como el gnosticismo y el maniqueísmo. Es también germen del cristianismo que, a través de algunos Padres de la Iglesia como Agustín de Hipona, Ambrosio de Milán, Isidoro de Sevilla o Gregorio de Tours, sentará las bases de la escolástica medieval. La belleza 'verdadera' se traslada entonces al mensaje y al espacio etéreo originado por la 'luz'4, más que al espacio físico de la arquitectura. La liturgia se convierte en la herramienta principal para transmitir la doctrina, ya que es capaz de generar espacios dinámicos alrededor de estructuras estáticas.

I Forman parte de un estudio más amplio que el aquí presentado, y todavía en proceso de elaboración. Me refiero a mi tesis doctoral: Arquitectura y construcción eclesiástica entre los siglos IV y X. Territorio de Empúries, y que está en su segundo año de desarrollo.

2 "Pues la belleza de las formas de la que yo ahora intento hablar no es lo que la mayoría supondría, por ejemplo la belleza de seres vivos o de ciertas pinturas, sino que me refiero a una línea recta o a una curva, afirma el argumento, y las superficies y sólidos derivados de ellas mediante tornos, reglas y escuadras, si es que lo entiendes. Y es que esas cosas afirmo que no son hermosas en relación con algo, como otras, sino que son por naturaleza siempre hermosas por sí mismas..." (Filebo, 5IC).

3 Estas valoraciones de Plotino sobre la belleza están recogidas en la p. 67 de la obra traducida por A. López y M. Tabuyo titulada Sobre la belleza. Este volumen trata específicamente dos fragmentos de las Eneadas, los relativos a la belleza.

4 Luz entendida en dos sentidos, el camino a la divinidad y la propia divinidad. Literalmente se habla de la belleza del alma. 
Si se analiza detenidamente la utilización de la geometría en la construcción, en primer lugar hay que valorar como se trasladarían las formas geométricas planteadas, a la práctica de los oficios relacionados con la edificación. Se debería hablar y de hecho así es, de dos tipos de geometrías, la teórica conceptual, y la práctica o fabrorum (Ruiz de la Rosa, 2005, p. IOOI). Platón, con una cierta ambigüedad, diferencia la geometría empírica de la teóricas , y sin embargo Vitruvio, que incluye en su desideratum una única geometría como conocimiento necesario del buen artifex ${ }^{6}$ utiliza el adjetivo fabrorum para diferenciar acciones arquitectónicas, pero no específicamente la geometría. Así Vitruvio distingue la arquitectura teórica o proyecto, ratiocinatio, de la práctica constructiva, fabrorum (I, I).

La geometría empírica proviene del mundo mesopotámico como constatamos en las tablillas cuneiformes con diagramas geométricos, provenientes de Nippur y Lagash (Rey, I943, p. 78) y datadas en el 3.000 a.C., así como la depositada en el Britisch Museum del I.700 a.C (Fig. I.I). Con posterioridad, la cultura egipcia aportó experiencia y observación al mundo griego, asentando los conocimientos a través de la escuela pitagórica. De hecho la geometría teórica de base científica reflexiva, proviene de la sistematización, también griega, de Euclídes. Sin duda Euclídes y Platón establecieron las bases de la llamada geometría de "regla y compás". Esta geometría aplicada con escuadra y compás, que utiliza también el agrimensor romano, ha sido documentada por la arqueología, gracias a la cultura material y la iconografía. Desde los primeros yacimientos pompeyanos a las múltiples estelas, el registro arqueológico presenta una variada tipología de instrumental (compases, escuadras, reglas, plomadas...) poniendo en evidencia su utilización tanto en la práctica del diseño como en la constructiva (Adam, 1996, p. 36 y 43).

Se afirma que el corpus geométrico aplicado a los oficios se mantendrá estable hasta el Renacimiento, mientras que paralelamente se seguirá avanzando en la resolución de problemas teóricos (Ruiz de la Rosa, 2005, p. I003). En este sentido es ejemplar contrastar la tablilla mesopotámica del I.700 a.C. (Fig. I.I) con la lámina 20 de Villard de Honnecourt (Fig. I.2), un maestro del siglo xiII que refleja en un cuaderno los conocimientos del oficio de la construcción. En ambos casos los esquemas para resolver de diferentes maneras la duplicación del cua-

5 “¿Y entonces? El arte del cálculo y el arte de la medida en la construcción y en el comercio, en comparación con la geometría filosófica y los cálculos teóricos... ¿̨hay que decir que cada una de esas artes es una o ponemos dos? Siguiendo con la argumentación precedente, yo, por mi parte, daría mi voto a que cada una de ellas es doble" (Filebo, 57a).

6 "El arquitecto debe ser un hombre letrado, experto en la realización de dibujos, erudito de la geometría, conocedor de muchas obras históricas, que hubiera escuchado con sumo interés a los filósofos, que supiera música, no desconocedor de la medicina, que supiera de jurisprudencia y que tuviera conocimiento de la astronomía y de sus leyes" (Vitruvio, I.I). 
drado son idénticos. Una de las soluciones la recoge también Platón en el diálogo de Menon (83a) ${ }^{7}$. Estos ejemplos tan distantes, hacen patente la continuidad en los conocimientos de la geometría aplicada. La geometría resuelve las diferentes cuestiones de forma más sencilla que la aritmética, dado que lo que se maneja son números irracionales. Una formalización geométrica ejemplar es la del anfiteatro romano, un triángulo, en unos casos equilátero en otro rectangular, genera la edificación (Gross, 200I, figs. 622 y 623).

Con el Imperio romano se normalizan dos elementos esenciales para la concepción del proyecto arquitectónico: la proporción y el módulo. Vitruvio, enuncia en el libro II las partes teóricas que componen la arquitectura: ordinatio, dispositio, eurythmia, symetria, distributio y decor ${ }^{8}$. En el libro IV al tratar de las casas particulares, argumenta que el primer paso del proyecto es determinar la regla de medidas a utilizar o sistema de proporciones; el segundo, trazar la planta para conocer su magnitud o determinar la anchura del espacio; y por último adaptar la proporción a la modulación de manera que inmediatamente salte a la vista la eurythmia.

Aunque, como atestigua Vitruvio, en la cultura griega ya se utilizaba el módulo, tanto el relativo al edificio ${ }^{9}$, como la unidad de magnitud ${ }^{\mathrm{Io}}$, será en época imperial cuando se codifica y expande el módulo como unidad de medida nor-

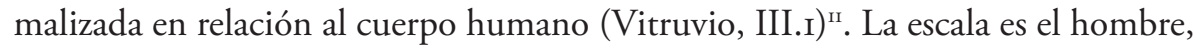
y desde el uncia y el digitus, hasta el decempedae, pasando por el palmus, el pes y el cubitus, el corpus de unidades de medida romanas queda perfectamente establecido y normalizado (Kurent, 1985). Pero estas magnitudes además de tener equivalencias entre ellas, están relacionadas geométricamente a través del triángulo pitagórico perfecto, el de lados 3, 4 y $5^{12}$, lo que facilita o estandariza las relaciones de proporción.

Para Vitruvio la simetrya es un concepto de orden que se rige por leyes geométricas, modulares o generalmente la combinación de ambas, lo que él llama rata pars, es decir la existencia de razones aritmético-geométricas en cada espacio y

7 Donde se refiere el conocido episodio en que Sócrates pregunta al esclavo como realizar la duplicación del cuadrado.

8 "Ordinatio es la ordenación de las partes respecto a un módulo del edificio, la fachada; dispositio es el proyecto o conjunto de planos e ideas con explicación geométrica; eurythmia es la armonía espacial surgida del correcto uso de la symetria; symetria es la combinación de las proporciones o razones tanto racionales como irracionales; distributio es la economía ajustada en el sentido de sostenibilidad actual; y decor entendido muchas veces como decoro, es la belleza ornamental adecuada a cada espacio teniendo en cuenta los ritos, tradiciones y costumbres" (Vitruvio, I.2). Ampliamente estudiadas por P. Gross o P. H. Shofield, o J. F. Esteban Lorente.

9 Diámetro mayor del fuste.

Io El pie ático.

II Existen precedentes por referencias como el 'canon de Policleto', en el campo de la escultura.

I2 Tres palmus son un dodran, cuatro un pes y cinco un palmipes. 
con el todo, o co-modulación. Así para la arquitectura vitruviana, la geometría es una herramienta que permite implantar las proporciones armónicas, que tanto preocupan a Vitruvio y a las que toda arquitectura debe aspirar, siempre teniendo como referencia la escala humana.

Una confirmación del manejo de la geometría aplicada a la concepción y diseño de la arquitectura durante los inicios de la Antigüedad Tardía, lo encontramos en la conocida epístola 25 del obispo Gregorio de Nisa, donde describe un edificio eclesiástico al que llama martyrion. La forma de la planta viene dada por la macla de circunferencias y rectángulos alrededor de una circunferencia central, es decir un diseño inicialmente geométrico que después toma dimensión con un único módulo director, la anchura de la nave ${ }^{13}$. Este canon se genera, en este caso, por una medición concreta en cubitus, pero la dimensión de la altura viene dada por la "proporción con la anchura". Gregorio de Nisa no necesita dar más que un único módulo con una magnitud concreta, a quién va dirigida la epístola también conoce las reglas del juego. El valor concreto que podía tener el cubitus lo ignoramos, pero está sin duda en relación directa con el módulo patrón que relaciona el diseño geométrico y las magnitudes.

Geometría, modulación y proporción forman una combinación indisoluble, y son conceptos esenciales para la generación del proyecto de arquitectura y, consecuentemente, para comprender el entorno histórico-cultural. Su diferente combinación, interpretación y aplicación facilitan una más amplia contextualización de la arquitectura como cultura material. Un ejercicio que he puesto en práctica con el análisis de la iglesia ampuritana de Santa Magdalena.

\section{SANTA MAGDALENA DE EMPÚRIES, UN EJEMPLO EN PROCESO DE ANÁLISIS}

El principal condicionamiento para analizar la concepción formal de un edificio, es el poder acotar sus fases crono-constructivas. La mayoría de edificios eclesiásticos han sufrido transformaciones a lo largo del tiempo, y cada una de estas fases puede responder a geometrías, módulos y proporciones diferentes. Solo el aislamiento de sus etapas, puede dar pie a su análisis con garantías de resultados

\footnotetext{
I3 "La dimensión en anchura de cada una de las salas rectangulares será de ocho codos, la longitud será algo más de uno y medio de ésta, pero la altura será la que requiera la proporción con la altura. Otro tanto ocurrirá con los absidiolos: el espacio entre las pilastras medirá igualmente ocho codos en total; y la medida que dé el trazado de un compás cuya punta va fijada en medio de éstos y que se hace pasar por sus extremidades, ésa será la profundidad. La altura será también la proporción con la anchura la que la determine. El espesor del muro que cierra exteriormente estos espacios interiores, según las medidas expuestas, será de tres pies y cerrará toda la construcción” (Teja, 1999, p. 69).
} 
fiables (Ripoll et al., 20I2, p. 62-64). Santa Magdalena d'Empúries situada cronológicamente a inicios de la horquilla estudiada, tiene bien establecidas sus fases crono-constructivas, razón por la que he escogido este yacimiento.

La pequeña iglesia ampuritana de Santa Magdalena se encuentra localizada junto a una vía secundaria de la red viaria romana (Bolòs y Hurtado, 2000) que conduce a San Martí d'Empúries, a poco más de un kilómetro de distancia y, a unos Ioom de Santa Margarida datada ya en el siglo x, pero edificada sobre estructuras anteriores.

Las últimas excavaciones llevadas a cabo en el yacimiento de Santa Magdalena documentaron la existencia de cuatro fases crono-constructivas (Aquilué y Nolla, 2003), pero la lectura de paramentos realizada por el actual equipo del Museu d'Empúries ha podido distinguir mejor las secuencias, especialmente entre la primera y segunda fase ${ }^{\mathrm{I}}$. La primera etapa, en el siglo IV, corresponde a un mausoleo de época romana bajo imperial, complejo de planta cuadrangular que aloja dos construcciones: la cámara funeraria cuadrada exteriormente con espacio interior octogonal, situada en el centro del perímetro lateral sur y un aula rectangular en la esquina noreste. La segunda etapa, en el siglo v, es la transformación del aula en iglesia cristiana, para ello se añade una cabecera también rectangular y un anexo en el paramento sur. La tercera y cuarta fase, en el siglo vi y x, son refacciones y ampliaciones del edificio eclesial y del conjunto. Se sustituye el ábside por uno cuadrangular abovedado y se completa la planta cruciforme. Finalmente se construye un campanario sobre el espacio presbiteral ${ }^{15}$.

El análisis que realizo muestra el diseño o proyecto geométrico del que parte el artifex. Son los esquemas geométrico-modulares que permitirán implantar el plan del edificio sobre el terreno para su construcción. Y los métodos de diseño que siguieron las sucesivas transformaciones a las que se somete el complejo edilicio.

En el proyecto inicial, la implantación del mausoleo, parece evidente que se delimite en primer lugar el terreno mediante un círculo, en este caso con un diámetro de 8 decempedae de casi 3,oom (Fig. 2a). La ocupación del conjunto está muy cercana al cuadrado inscrito en esta circunferencia, con sus lados orientados a los cuatro puntos cardinales, y la dimensión del ancho del aula es la resultante de la operación geométrica de superponer este cuadrado y el triángulo equilátero

I4 Quiero agradecer a M. Santos, Q. Tremoleda y P. Castanyer las aclaraciones que me suministraron en referencia a la lectura de paramentos de Santa Magdalena, especialmente teniendo en cuenta que dichos estudios están a día de hoy inéditos. Este texto se ha visto enriquecido por su generosa información.

I5 Estas fases están grafiadas en la planta de la Fig. 2, siguiendo el protocolo diseñado para el CARE (Corpus de architecturae religiosae europeae, saec. $V-X$ ). 
inscrito también en la misma circunferencia (intersección del lado del cuadrado 2-3 y el lado del triángulo equilátero I-3 en la Fig. 2b). Esta dimensión de I4 cubitus de $0,44 \mathrm{~cm}$ (el pes correspondiente es el de $0,29 \mathrm{~cm}$ ), es el lado del cuadrado que genera al doblarse la superficie del aula y origina también la planta del edificio principal. Este edificio con planta interior octogonal y exterior cuadrada se emplaza teniendo como uno de sus ejes el diámetro mayor N-S, y a partir de un punto central localizado por la traslación del lado del cuadrado (segmento 2-3 = segmento $2-4$ en la Fig. 2b) inscrito en la circunferencia delimitadora, desde el extremo norte de éste diámetro (centro 2), tal como se describe en la figura 2 (Fig. $2 \mathrm{a}, 2 \mathrm{~b}, 2 \mathrm{c}$ y $2 \mathrm{~d})$.

Estas operaciones geométricas, de sencilla aplicación en el terreno, siguen una línea teórica que proviene de la cultura griega. Para Platón en Timeo, el mundo se concentra en una esfera, el cuerpo más perfecto por la igualdad de distancia del centro a cualquier punto del perímetro. Y el cubo es el sólido que compone la tierra, elemento más pesado que los compuestos con triángulos: fuego, aire y agua. Las proporciones en esta fase de Santa Magdalena se regulan en base a módulos romanos (decempedae, cubitus y pes) conjugados con sencillas razones como la duplicación (razón $=2$ ) en el caso del aula, o la utilización del lado de polígonos regulares inscritos en la circunferencia (cuadrado y triángulo equilátero), según conceptos vitruvianos de co-modulación.

En la segunda fase se produce un cambio de uso. El espacio del aula se adapta y transforma para uso litúrgico cristiano. Se trata de una planta preexistente, es decir que tanto la geometría como la modulación tendrán como punto de partida magnitudes y formas heredadas de la fase anterior. Sin embargo hay una pequeña variación en estas dimensiones ya que se trasdosan los muros oeste y sur de este espacio, con un aumento de grosor de unos $15 \mathrm{~cm}$ que se acerca al semis romano ${ }^{16}$, tal como ha constatado el equipo del Museu d'Empúries. No es el momento de entrar en las razones constructivas en cuanto a la finalidad de esta obra o reparación, pero sí en la implicación en cuanto al cambio de su dimensionado. ¿¿Se modifica la modulación de este espacio? En primer lugar el calibrado del muro es irregular, realizado con aparejo de piedra poco trabajada y que ha perdido una capa de revoco. Es decir que la medida de su grosor en los diferentes puntos del mismo, nunca da un resultado exacto, por lo que se debe considerar un margen de variación de unos $6-8 \mathrm{~cm}$, dimensión que ya equivale a un palmus romano. En segundo lugar, la correspondencia entre las numerosas medidas romanas, considero que en el contexto del proceso constructivo es una característica más susceptible de ser utilizada en la práctica que la propia dimensión exacta. En consecuencia sigo estimando que la dimensión del aula es de i4 cubitus, pero que

I6 El semis es equivalente a 2 palmus romanos de $7,39 \mathrm{~cm}$. 
han pasado a tener una magnitud de $45,3 \mathrm{~cm}$ en lugar de los $44,36 \mathrm{~cm}$ canónicos romanos. El diseño general se basará en la utilización de la geometría aplicada cuya base modular es un elemento del edificio, en este caso el ancho del espacio (I4 cubitos), más que en la aplicación de magnitudes normalizadas.

La longitud de la nueva iglesia queda definida trazando una circunferencia cuyo radio es la diagonal del cuadrado que tiene por lado el ancho externo del aula. El centro de esta circunferencia se coloca en el eje longitudinal del aula a la distancia marcada por el radio. Quedan definidas de esta forma, las dimensiones máximas de la iglesia que incluyen el nuevo ábside y el espacio adosado al sur (Fig. 3a y 3b). Esta ubicación de la circunferencia, no es aleatoria, su centro se distancia del centro de la primera circunferencia de delimitación del terreno para el mausoleo, exactamente el ancho del aula o nave de la nueva iglesia (Fig. 3c). La razón que rige ahora es $\sqrt{2}$, derivada de las propiedades geométricas del nuevo trazado $^{17}$. Una vez delimitada la nueva cabecera y la longitud de la nave, para acabar de acotar la ampliación del volumen al sur, se dibuja la misma circunferencia con centro en el punto medio del muro exterior de la cabecera. Con esta geometría se obtendrá tanto el ancho como el largo del nuevo cuerpo lateral, coincidiendo por sus propiedades geométricas intrínsecas, con la delimitación del presbiterio, cuya parte inferior se corresponde con el centro del nuevo conjunto (Fig. 3b), se trata de una vesica piscis (mandorla).

En el siglo VI se construye un nuevo ábside, quizá por deterioro del anterior o simplemente se reduce la luz para abovedarlo. Además se completa la simetría de la planta cruciforme ańadiendo un cuerpo al norte. Se trata de remodelaciones y ampliaciones sin aparente cambio de uso, no parece justificado un nuevo diseño geométrico, ya que puede copiarse la planta existente en el caso del cuerpo añadido, y trasdosar los muros del ábside. Sin embargo el espacio interior absidial mide $7,5 \times 8,5$ cubitus de $0,45 \mathrm{~cm}$, y el triángulo equilátero inscrito en la circunferencia que da origen al edificio de la primera iglesia, marca un punto significativo: el interior norte del inicio del ábside. Es probable que no exista una intencionalidad en este punto, ya que puede derivarse de las propiedades intrínsecas de la geometría original, pero el análisis de la planta y del alzado interior del ábside plantea dudas, y permite observar coincidencias. La planta responde al cuadrado cuyo lado es el ancho interior del nuevo ábside más un tercio del radio (x) de su circunferencia inscrita o, si incluimos el grosor del muro, un tercio del diámetro (y) de la misma (Fig. 4a). Por lo tanto, el nuevo espacio interior responde a la razón armónica de 4/3 = I,333. Asimismo al conservarse el arranque de la bóveda se ha podido estudiar el alzado y redibujar la sección interna del ábside. La altura

I7 He dicho que el radio de esta circunferencia era la diagonal del cuadrado y por tanto su razón es $\sqrt{2}$. 
en el punto máximo es el ancho del ábside 3,3m, ligeramente superior a la mitad de la anchura de la nave $(6,34 \mathrm{~m})$. Insertando un triángulo rectángulo perfecto $(3$, $4,5)$ y colocando la hipotenusa perpendicular al plano del pavimento, se observan dos circunstancias. La primera es que el ángulo recto del triángulo está marcando la línea donde se encuentran los mechinales (I,2O-I,3Om) utilizados para la construcción de la bóveda. La segunda es que el módulo básico del triángulo es de $0,67 \mathrm{~cm}$, es decir I, 5 cubitus de $0,45 \mathrm{~cm}$ (Fig. 4b).

También es significativa la última actuación que se hace en la iglesia durante el siglo X, cuando se añade un campanario sobre el presbiterio, similar a lo que ocurre en Santa Helena de Rodes. Para ello se doblan los muros interiores del espacio presbiteral, obteniéndose una planta donde se encaja un rectángulo que tiene como razón de semejanza entre sus lados la $\sqrt{2}$, y cuyas dimensiones son de 9,5 x 6,75 cubitus de 0,45cm (Fig. 4, Fase 4).

El cambio dimensional de los módulos normalizados romanos, es inapreciable, la variación se ha producido en la tipología de la geometría utilizada. La siguiente tabla resume las dimensiones de los módulos y la razón empleada en cada una de las fases.

\begin{tabular}{l|l|l|l|l} 
& FASE I & FASE II & FASE III & FASE IV \\
\hline PES & 0,2953 & 0,301 & 0,301 & 0,301 \\
\hline CUBITUS & 0,443 & 0,45 & 0,45 & 0,45 \\
\hline DECEMPEDAE & 2,953 & 3, OI & 3, OI & 3, OI \\
\hline MÓDULO & Lado cuadrado & Ancho nave & & \\
\hline RAZÓN & 2 & $\sqrt{2}$ & $4 / 3$ & $\sqrt{2}$
\end{tabular}

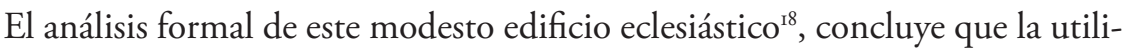
zación de la geometría aplicada y la proporción regida por módulos conocidos, no es debida al azar o a la simple casualidad. Las propiedades de la primera geometría quedan asumidas en las nuevas formas con una cierta inconsciencia, no obstante es evidente que la intencionalidad de modelos geométricos y razones proporcionales conocidas, muestran las prácticas utilizadas.

\section{TRANSLATIO O TRANSFORMACIÓN: CONCLUSIONES}

En la carta de Gregorio de Nisa se encuentran todavía los códigos vitruvianos en cuanto a la utilización de la geometría, la modulación, y la proporción, aunque

I8 Santa Magdalena d'Empúries tiene una superficie total de 165m2 construidos. 
no podamos constatar la dimensión de las magnitudes utilizadas. En Santa Magdalena se comprueba como el empleo de la geometría, el código de magnitudes y la utilización de leyes de proporcionalidad conocidas, se mantienen a lo largo de las diferentes fases crono-constructivas. En ambos casos se están empleando las mismas herramientas conceptuales y metodológicas para el diseño de la arquitectura.

Cabe destacar que el hecho de que los conocimientos de la geometría aplicada se perpetúen, no significa que la utilización de las proporciones o razón vaya a ser siempre la misma. La variación puede estar relacionada con múltiples causas, desde la significación o códigos que se otorgan a cada uso del espacio, hasta las tradiciones y experiencia de cada uno de los talleres o artifices.

Por último es necesario resaltar que si en el siglo XIII, Villard de Honnecourt evidencia que la geometría aplicada se sigue utilizando, no se puede confirmar por sus notas, que suceda lo mismo con los módulos y las leyes de proporcionalidad. En conclusión los diferentes artifices, aplican unos conocimientos técnicogeométricos que, fruto de la translatio, se difunden tanto en el espacio como en el tiempo, desde el mundo greco-romano al mundo medieval, desde Vitruvio a Villard de Honnecourt.

\section{BIBLIOGRAFÍA}

Adam, J.-P., 1996: La construcción romana. Materiales y técnicas, León.

Aquilué Abadias, X., y Nolla Brufau, JM., 2003: Memòria de les excavacions arqueològiques efectuades l'any 2003 a les Esglèsies de Santa Margarida i Santa Magdalena d'Empúries. Memória inédita. Para su consulta: Dipòsit Digital del Departament de Cultura (CALAIX), <http://calaix.gencat.cat/ handle/Io687/9415?>.

Bolòs, J., y Hurtado, V., 2000: Atles del Comtat d'Empúries-Peralada, Rafael Dalmau ed., Barcelona.

Esteban Lorente, J. F., 200I: "La teoría de la proporción arquitectónica en Vitruvio", Artigrama, I6, pp. 229-256.

Gross, P., 2006: Vitruve et la tradition des tratités d'architecture. Fabrica et ratiocinatio, Recueil d'études, Roma.

Kurent, T., I985: "La coordinación modular de las dimensiones arquitectónicas", Boletín del Museo Arqueológico Nacional, III, Madrid, pp. 69-94.

Platón, Menón, introducción y edición de S. Vegas González, traducción de A. Ruiz de Elvira, Madrid, 2oIo. 
—, Filebo, introducción de J. M. Aguado Rebollo, traducción y notas de E. Sánchez Millán, Madrid, 20II.

—, Timeo, introducción y traducción de F. Lisi, Madrid, 2oII.

Plotino, Sobre la belleza, prólogo de Agustín López, traducción de Agustín López y María Tabuyo, Barcelona, 2007.

Rey, A., 1943: La ciencia oriental antes de los griegos, París.

Ripoll, G., Carrero, E., Rico, D., Tuset, F., Velázquez, I., López Batlle, A., Mas, C., Valls, M., y Cau, M.Á, 20I2: "La arquitectura religiosa hispánica del siglo IV al X y el proyecto del Corpus Arqchitecturae Religiosae Europeae - CareHispania”, Hortus Artium Medievalium, I8/I, pp. 45-73.

Ruíz de la Rosa, J. A., 2005: "Fuentes para el estudio de la geometría fabrorum. Análisis de documentos", en Actas del Cuarto Congreso de Historia de la Construcción, ed. S. Huerta, Cádiz, pp. IOOI-Ioo8.

Shofield, P. H., 1971: Teoría de la proporción en arquitectura, Barcelona.

Teja, R., 199I: "Gregorio de Nisa arquitecto y empresario: Epístola 25", Arte, sociedad, economía y religión durante el Bajo Imperio y la Antigüedad Tardia, Antigüedad y Cristianismo, VIII, Murcia, <http://revistas.um.es/ayc/article/ view/6236r>.

Vitruvio, Los diez libros de Arquitectura, versión de J. L. Oliver Domingo, Madrid, 1995 (reimpresión 20II). 


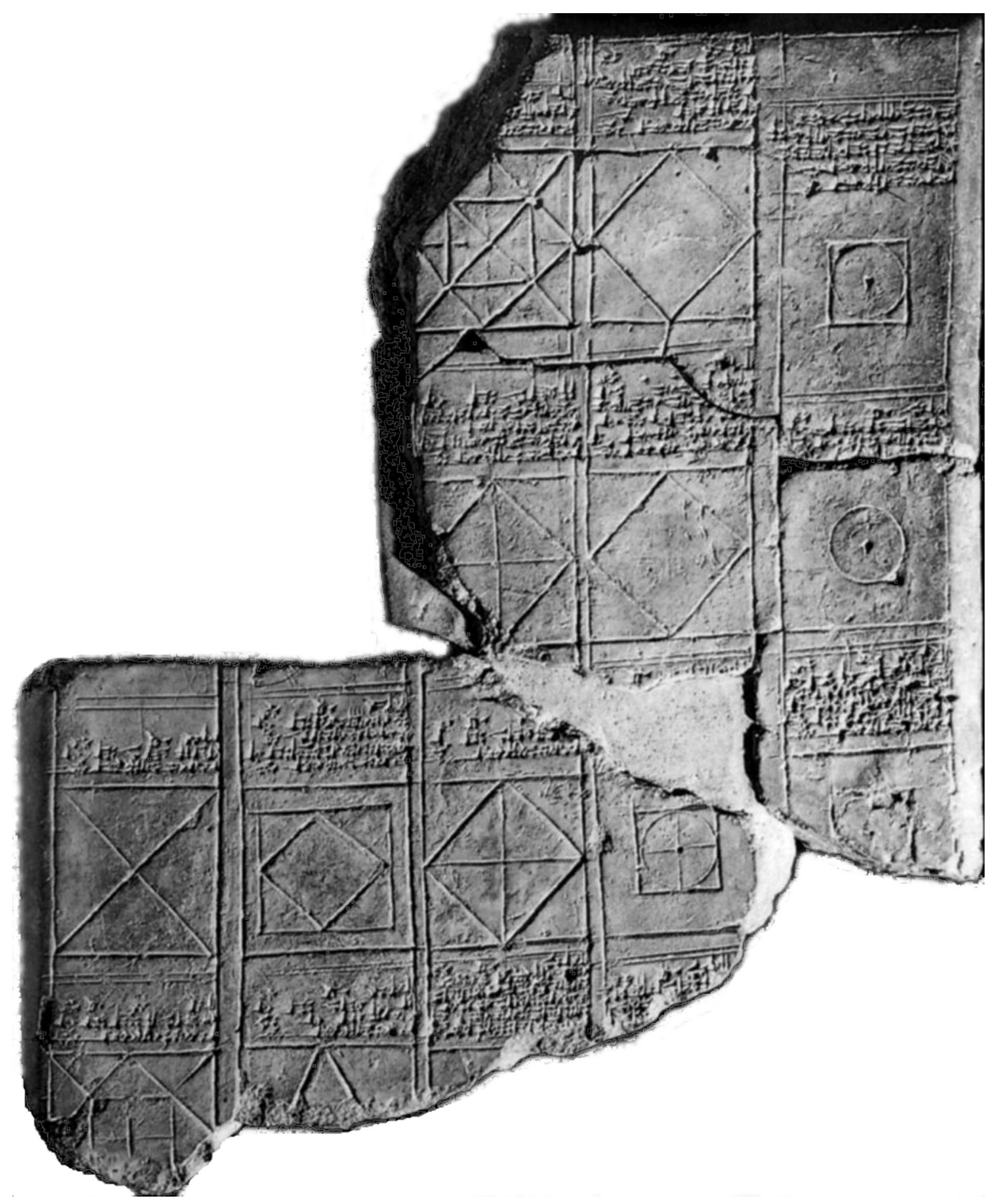

Fig. I.I. Tablilla cuneiforme mesopotámica del I.700 a.C. (British Museum). 


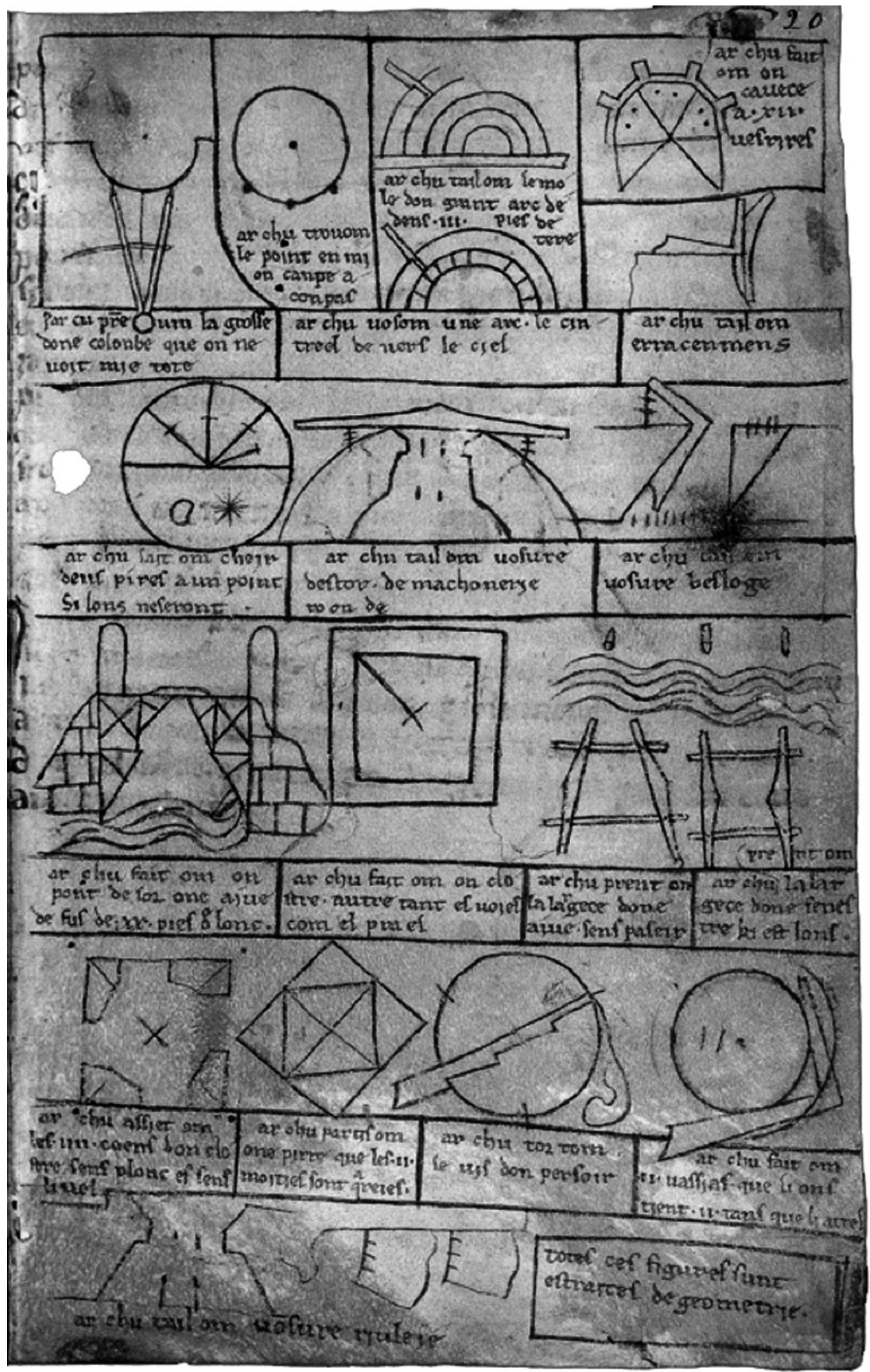

Fig. I.2. Lámina 20 del cuaderno de Villard de Honnecourt. 
Planta Santa Magdalena d'Empúries
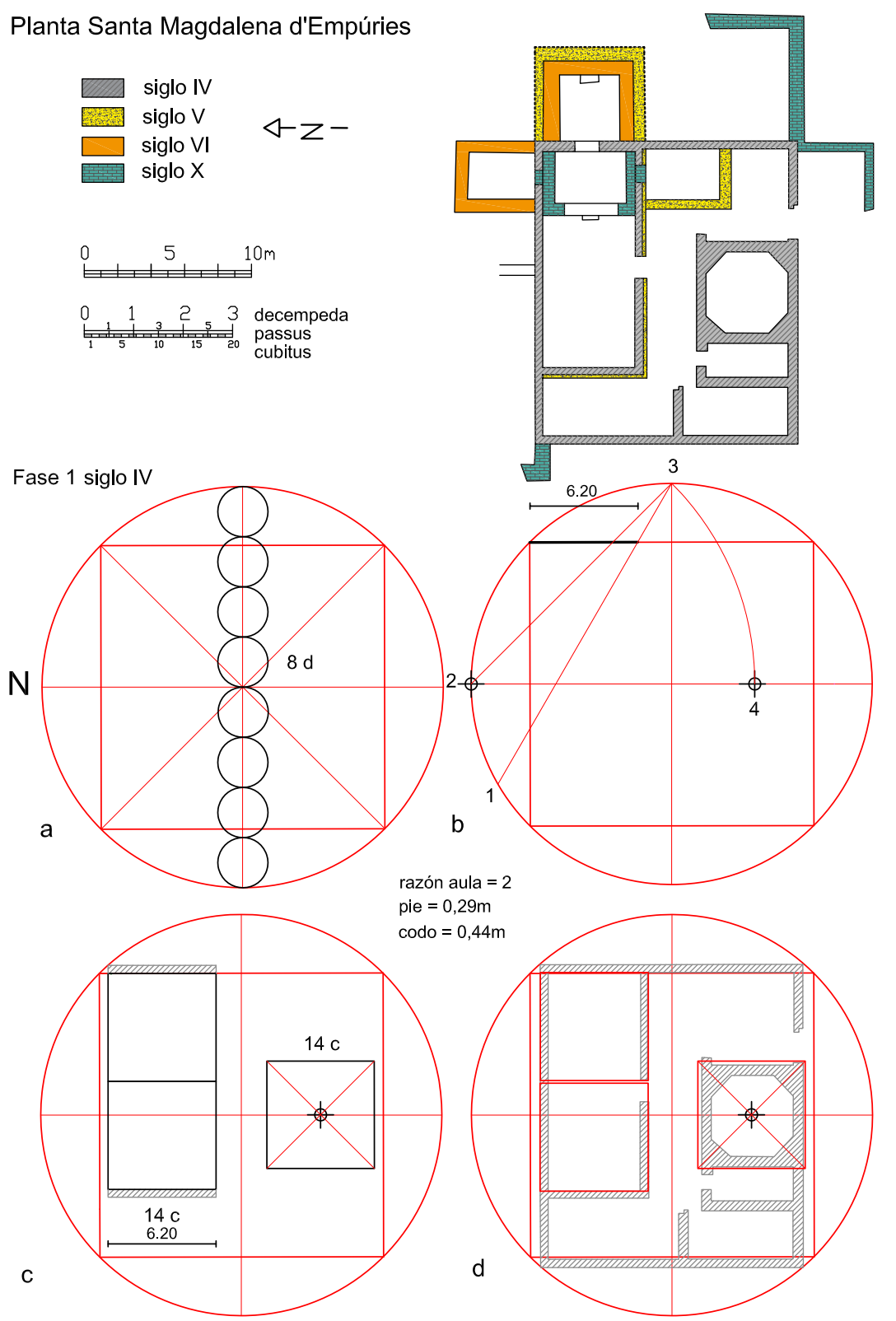

Fig. 2. Planta de Santa Magdalena d'Empúries, fases crono-cronstructivas (CARE) (dibujos de la autora): 2a Delimitación del terreno en fase I (siglo Iv); 2b Delimitación de la anchura y la longitud del aula, y centro del edificio octogonal en fase I; $2 \mathrm{c}$ Diseño de la planta en base al cuadrado, y medidas de los módulos utilizados en el aula en fase I; $2 \mathrm{~d}$ Modulación de todo el complejo en fase I. 


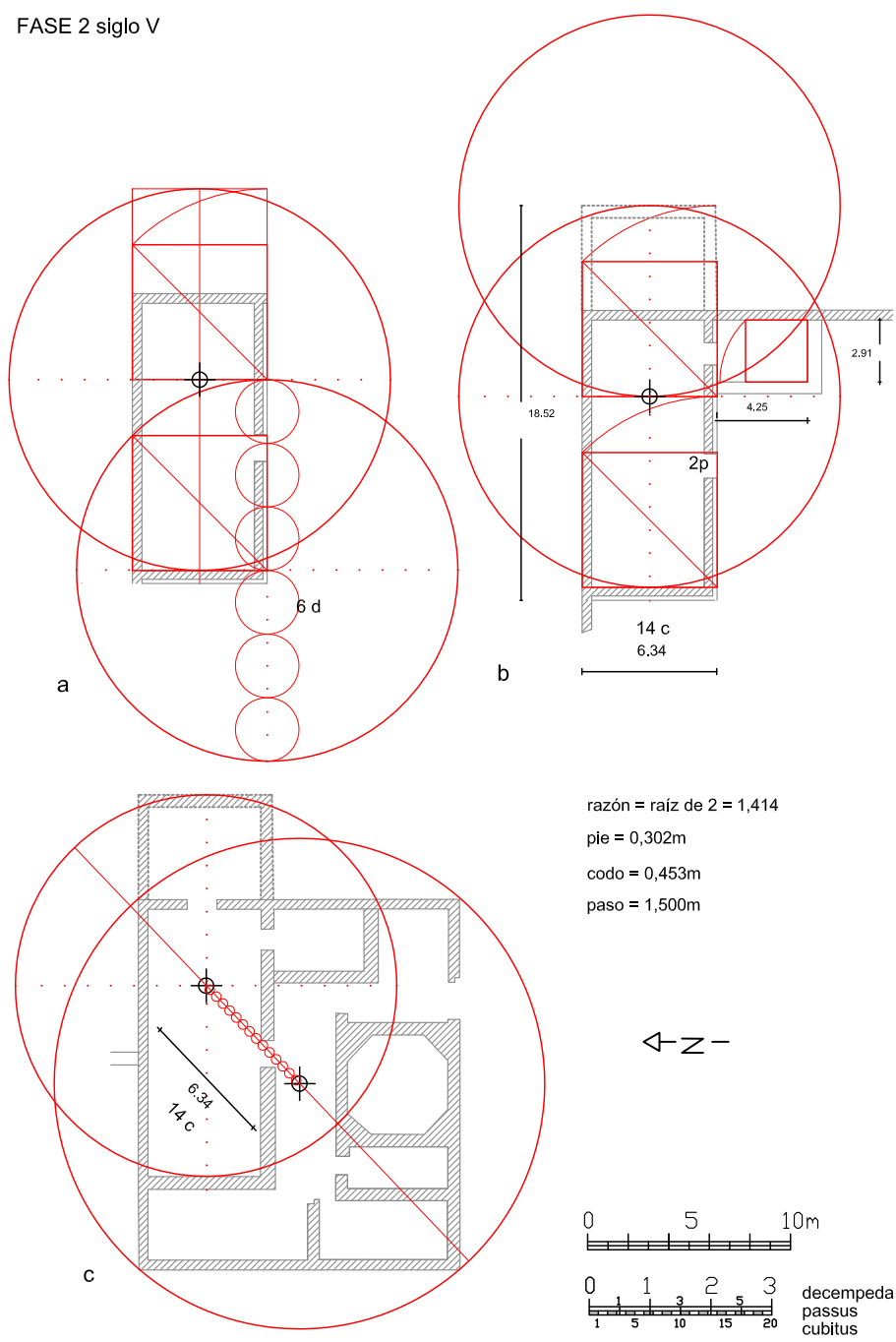

Fig. 3. Santa Magdalena d'Empúries fase 2 (siglo v) (dibujos de la autora): za Geometría origen de las nuevas proporciones; 3 b Diseño de la primera planta de la iglesia; $3 \mathrm{C}$ Relación entre fase I y 2. 
FASE 3 siglo VI
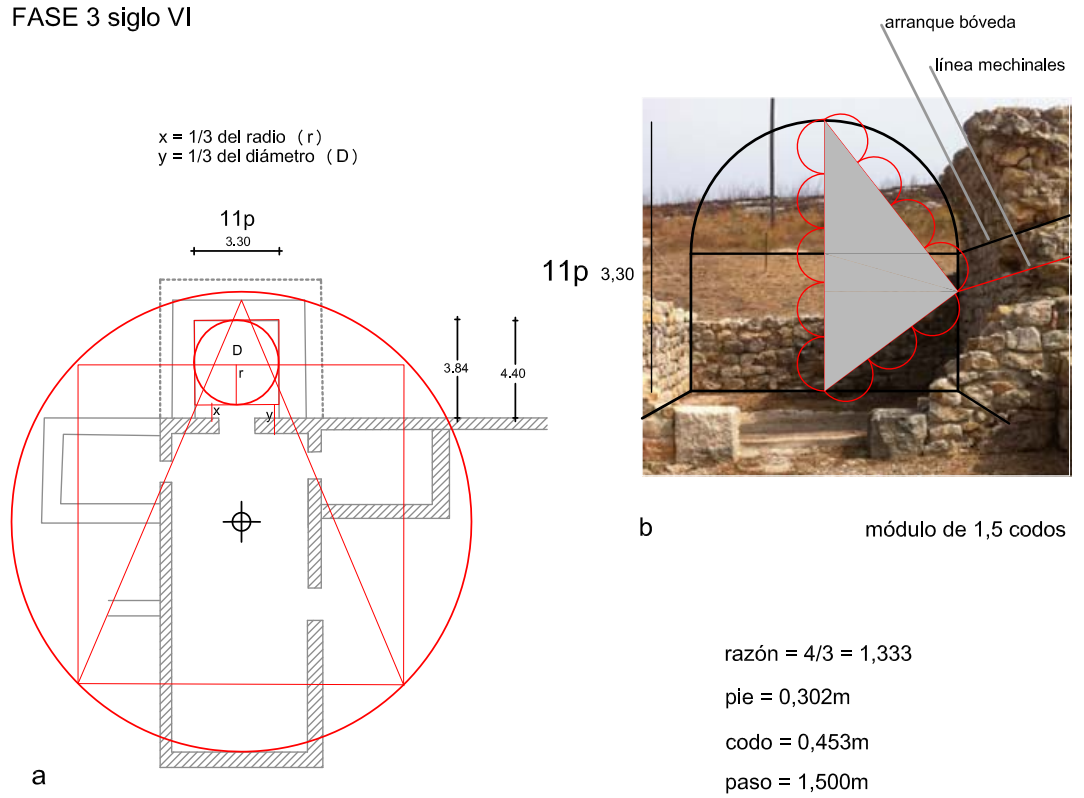

FASE 4 siglo $\mathrm{X}$

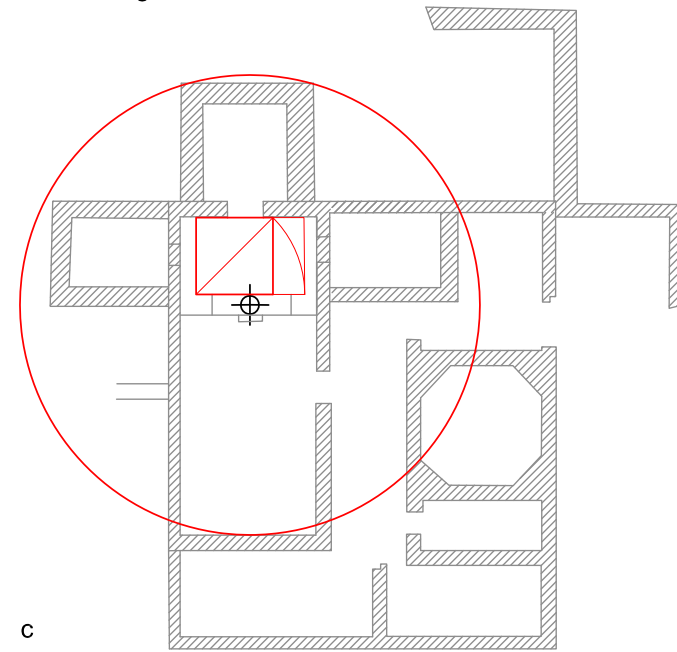

razón $=$ raíz de $2=1,414$
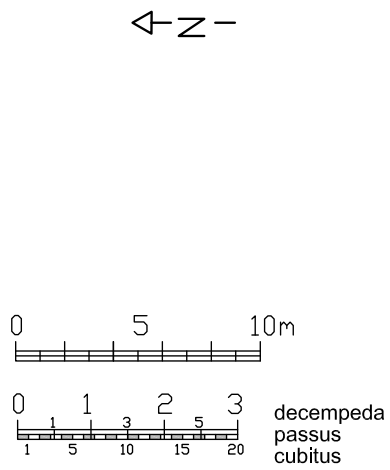

Fig. 4. Santa Magdalena d'Empúries fases 3 (siglo vi) y 4 (siglo x) (fotografía y dibujos de la autora): 4a Geometría y módulos en la planta del siglo vi; 4 b Esquema de alzado interior del ábside del siglo vi sobre fotografía actual; 4c Santa Magdalena d’Empúries fase 4 (siglo x): geometría y modulación. 